\title{
Cardiac tamponade as the initial manifestation of pulmonary adenocarcinoma
}

\author{
Mitja Letonja ${ }^{1}$ and Andrej Debeljak ${ }^{2}$ \\ ${ }^{1}$ Department of Internal Medicine, General Hospital Ptuj, ${ }^{2}$ University Clinic of Respiratory and \\ Allergic Diseases Golnik, Slovenia
}

\begin{abstract}
Background. Neoplastic pericarditis can be presented as acute pericarditis, pericardial effusion, effusiveconstrictive pericarditis or cardiac tamponade. For the majority of patients, a clinical manifestation of neoplastic pericarditis is absent or remains unrecognised during their life.

Case report. A 69-year-old non-smoking woman with acute dyspnoea, tachycardia, jugular venous distension, hepatomegaly and right side pleural effusion was presented in the emergency department. The roentgen picture of the chest confirmed pleural effusion and revealed enlarged heart. An emergency echocardiogram established the diagnosis of cardiac tamponade. The patient improved immediately after the therapeutic pericardiocentesis. Cytology of pericardial fluid confirmed malignant glandular cells, consistent with metastatic adenocarcinoma. A computed chest tomography showed a right side pleural effusion and a solitary round lesion in the right lower lobe of the lung. Bronchoscopy with fundoscopic lung biopsy and brushing revealed adenocarcinoma.

Conclusions. Cardiac tamponade as the initial manifestation of malignancy is rare and rare is also malignant pericardial effusion due the adenocarcinoma of the lung in a non-smoking woman. The presented patient had one of the longest survival reported in literature despite a limited life expectancy regardless of the treatment in the patient presented with neoplastic pericarditis.
\end{abstract}

Key words: lung neoplasms; adenocarcinoma; cardiac tamponade; pericardial effusion

\section{Introduction}

In necropsy series, the pericardium is involved in 5 to $15 \%$ of patients with a malignant disease. ${ }^{1}$ Neoplastic pericarditis can be presented as acute pericarditis,

Received 30 August 2007

Accepted 9 September 2007

Correspondence to: Mitja Letonja, M.D., Ph. D.; General Hospital Ptuj, Department of Internal Medicine, Potrčeva 23-25, 2250 Ptuj, Slovenia; Phone: +386-2-7462401;; E-mail: mitja.letonja@mf.uni-lj.si pericardial effusion, effusive-constrictive pericarditis or cardiac tamponade. ${ }^{2}$ For the majority of patients, a clinical manifestation of neoplastic pericarditis is absent or remains unrecognised during their life. Cardiac tamponade as the initial manifestation of a malignancy is rare and such patients have a very limited life expectancy. ${ }^{3}$ Here we reported a 69-year-old woman with clinical signs of cardiac tamponade as the initial presentation of lung cancer. The literature has been reviewed at length. 


\section{Case Report}

A 69 year-old woman was admitted to our hospital because of acute worsening of dyspnoea. She had a 4 months history of exertional dyspnoea, weight loss of $5 \mathrm{~kg}$, anorexia and chest pain. She had never smoked. The physical examination showed blood pressure 150/90 $\mathrm{mmHg}$, pulse 122 beats/min, respiration $28 / \mathrm{min}$ and temperature $37.4{ }^{\circ} \mathrm{C}$. The jugular vein was distended to the angle of the jaw. The chest was dull to percussion on the basal part on the right side. The apex beat was not palpable and the area of cardiac dullness was increased. The heart sounds were faintly audible. The liver was $5 \mathrm{~cm}$ below the coastal margin and it was tender.

Her hematologic and biochemical values were normal except for the elevated sedimentation rate $(35 \mathrm{~mm} / \mathrm{h})$. The chest $\mathrm{x}$-ray showed a moderate cardiac enlargement and a right side pleural effusion. The electrocardiogram showed a right bundle branch block. Echocardiography confirmed a large pericardial effusion with the evidence of diastolic collapse of the right atrium and right ventricle, indicating cardiac tamponade.

The indwelling pericardial catheter was inserted into the pericardial cavity with a subxiphoid access and drainage of 1100 $\mathrm{ml}$ haemorrhagic fluid was done (Figure 1). The duration of drainage was 3.8 days.

We instilled $8 \mathrm{mg}$ of dexamethasone into the pericardium before we removed the catheter. Cytology of pericardial fluid confirmed malignant glandular cells, consistent with metastatic adenocarcinoma. Bacterial and acid fast cultures of pericardial fluid were negative. Immediately after the pericardiocentesis the patient clinically improved. CT showed a right side pleural effusion and a solitary round lesion in the right lower lobe of the lung (Figure 2). Bronchoscopy with bronchscopic lung biopsy and brushing under fluoroscopic

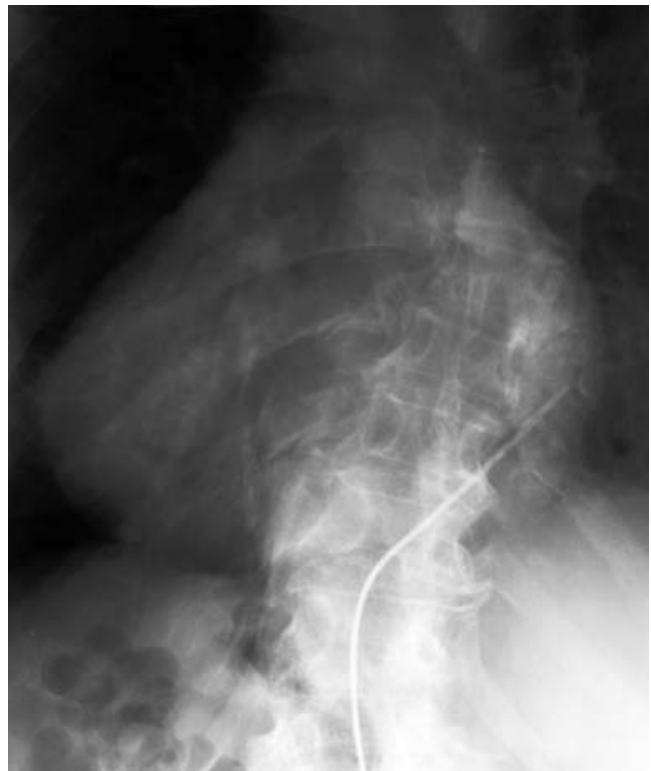

Figure 1. Radiograph of the heart with indwelling pericardial catheter in pericardial cavity.

guidance revealed adenocarcinoma. The diagnosis of the malignant right side pleural effusion was made by pleural biopsy. Over the next 30 months she developed recurrent, large, symptomatic, malignant right side pleural effusion that was treated with numerous therapeutic thoracentesis and talc pleurodesis.

Fifteen months after the initial presentation, the patient suffered from the left hip pain due to an osteolytic lesion in osis ilei in the left osis pubis for which she underwent a radiation therapy of the left side of pelvis and we advised the patient to unload her left leg.

After 26 months we found left side pleural effusion and adenocarcinoma cells in the left pleural fluid. We treated the patient during 14 days with $16 \mathrm{mg}$ methylprednisolone every second day and then for one weak with $8 \mathrm{mg}$ methylprednisolone every second day.

Twenty-seven months after the initial presentation, the patient received one course of chemotherapy consisting of the 


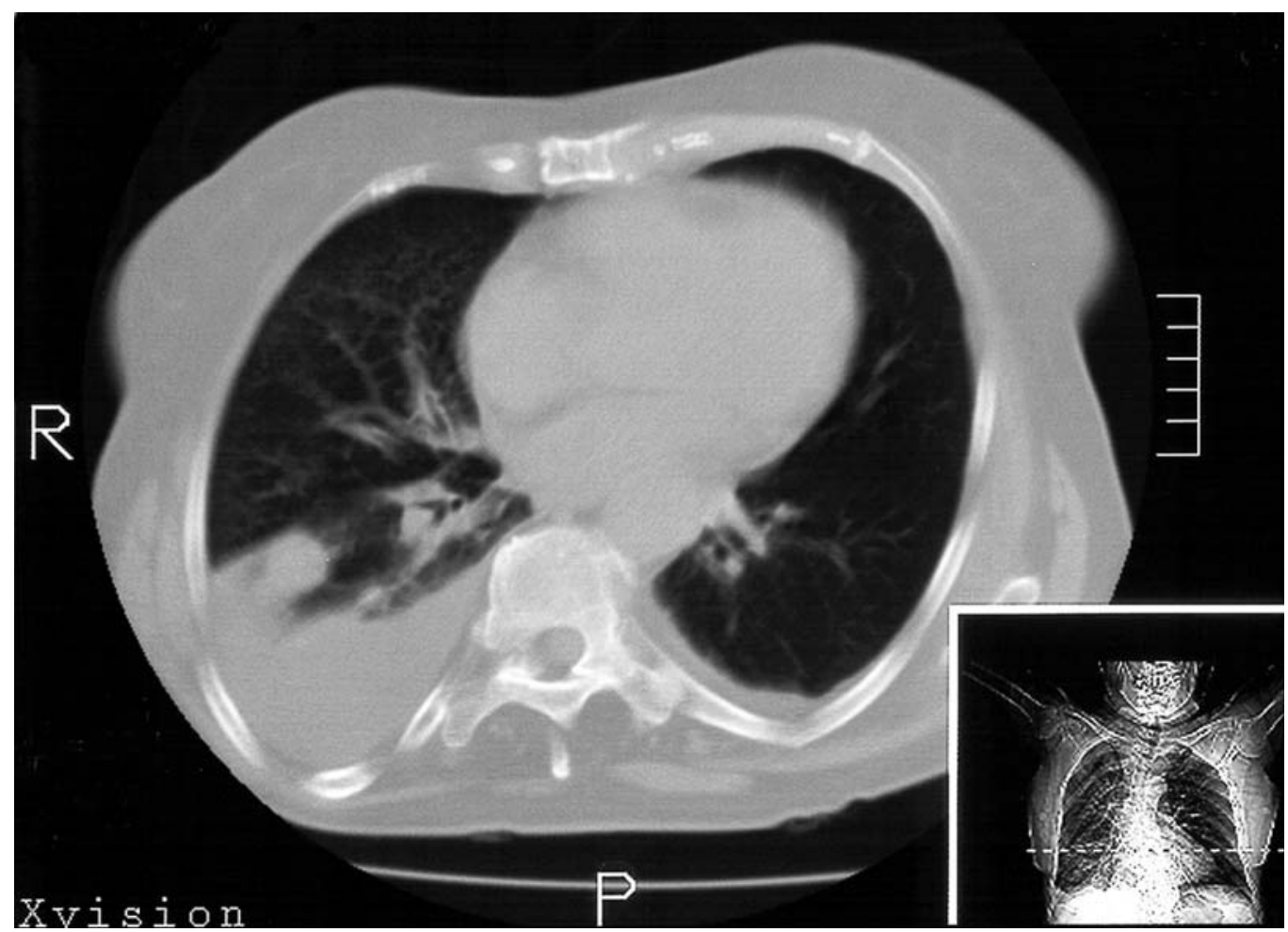

Figure 2. CT scan of the chest with right side pleural effusion and solitary round lesion $2 \mathrm{~cm}$ in diameter located in posterior basal segment of the right lower lobe of the lung

combination of cisplatin $160 \mathrm{mg}$ and etoposide $500 \mathrm{mg}$ in three consequent days with $10 \%$ mannitol, dexamethasone and tropisetron. At the end of chemotherapy the patient felt the progression of profound weakness, nausea and vomitus, which required hospitalisation. We discovered pancytopenia: haemoglobin $10.1 \mathrm{~g} / \mathrm{dL}$; white blood cells $0.7 \times 10^{3} / \mu \mathrm{L}$; neutrophils $7 \%$; stabs $9 \%$; lymphocytes 60\%; eosinophils 3\%; monocytes $20 \%$; and platelets $79 \times 10^{3} / \mu \mathrm{L}$. Neutropenia was prolonged and because of the appearance of subfebrile temperature, we administrated granulocyte colony-stimulating factor filgrastim, antibiotic and antimicotic and the patient improved. Because of a febrile neutropenia occurring after the first chemotherapy, the patient did not receive a second course.
Twenty-nine months after the initial presentation the patient became occasionally confused and CT of the brain detected metastatic lesions from $2 \mathrm{~cm}$ to $0.5 \mathrm{~cm}$ in diameter which enhanced after the application of intravenous contrast.

During the illness we repeated echocardiography several times and find thicker pericardium without significant pericardial effusion. The patient died 30 months and 1 week after the diagnosis of malignant pericardial effusion was made.

\section{Discussion}

The first comprehensive review of 29 isolated cases of pericardial effusion as the initial manifestation of malignancy was 
published by Fraser in $1974 .{ }^{3}$ After that Fincher reviewed 61 cases of metastatic cancer presenting as malignant pericardial effusion from 1974-1991. ${ }^{4}$ Reports of cardiac tamponade as a initial presentation of malignancy were not so common as the reports of pericardial effusion, but there is a doubt if the occurrence of malignant cardiac tamponade is underestimated because of non-specific signs and symptoms. ${ }^{5}$

Our patient presented with acute dyspnea which is also the most common presenting symptom in a review of malignant tamponade pooling several series with incidence of $78 \%$. The other reported symptoms were cough $(46 \%)$, chest pain $(27 \%)$, orthopnea $(26 \%)$, and weakness (19\%). On the physical examination the most frequently detected findings are sinus tachycardia (50\%), jugular venous distention (45\%), hepatomegaly (36\%) and peripheral oedema (35\%). Classical findings of pericardial disease such as pulsus paradoxus, pericardial rub and Kussmaul's sign - occurred in only $30 \%, 12 \%$ and $5 \%$, respectively. ${ }^{6}$ Because of non-specific symptoms and signs, a clinical recognition of pericardial involvement in patients with malignancy is rare.

ECG changes such as electrical alternans and other were not presented in our case and they are neither common nor specific findings for malignant pericardial effusion. ${ }^{2}$ A chest $x$-ray showed non-specific cardiomegaly. Pleural effusion was presented in our patient and in more than in half of the patients in literature. ${ }^{2}$ The echocardiogram documented the presence and magnitude of pericardial effusion and differentiates cardiac tamponade from other causes of systemic venous hypertension and arterial hypotension, including constrictive pericarditis, cardiomyopathy and right ventricular infarction. $^{7}$

The cytological examination of pericardial fluid confirmed the diagnosis of malignant pericardial effusion in $65 \%$ to $85 \%$ of cases. ${ }^{8}$ An open pericardial biopsy can identify most of the remaining cases. ${ }^{2} \mathrm{~A}$ search for primary tumour must be undertaken because metastases to the pericardium are much more common than primary tumour. The underlying malignancy is most often carcinoma of the lung (40\%), breast (22\%), leukemia and lymphoma (15\%), sarcomas $(3.5 \%)$ and melanoma $(2.7 \%) .{ }^{9}$ There are also other causes of pericardial effusion in patients with cancer: radiation-induced, idiopathic, drug-related or uremic. ${ }^{2}$

Cardiac tamponade requires an urgent drainage of fluid. It is effective in $90 \%$ of patients, with $1 \%$ of major complications. The majority of authors instilled cytostatics (bleomycin, cisplatin and thiotepa) or nonspecific sclerosing agents (tetracyclines, dexamethasone) into pericardium to prevent recurrence. ${ }^{10-13}$ We also instilled dexsamethasone, but numbers of such treated patient is too small to estimate the efficacy and safety of these agents. We also do not know the spontaneous re-accumulation rate of malignant effusion after pericardiocentesis because a number of patients reported in literature not receiving any additional therapy is too small. ${ }^{10-13}$ We treated our patient with systemic chemotherapy late in the course of the illness. Systemic chemotherapy and radiation therapy are successfully used in breast cancer, and leukemia and lymphoma after the initial pericardiocentesis to prevent the recurrence of effusion and to treat primary cancer. ${ }^{10,11}$ In cases of recurrent effusion and persistent symptoms various surgical drainage procedures are available. A total pericardiectomy is seldom performed today for pericardial effusions associated with malignancy because the operative risks are too high. Recent literature favours the creation of a pericardial window either by thoracotomy, by a subxiphoid route, or by thoracoscopy. ${ }^{10}$

We describe a case of malignant pericardial effusion as the initial presentation of 
adenocarcinoma of the lung that has several diversities from previously reported patients with malignant cardiac tamponade. Our patient has never smoked and she developed lung carcinoma. Most female patients presented with cardiac tamponade had underlying breast carcinoma and our patient survived 30 months after the diagnosis while the average survival rate of patients with cardiac tamponade secondary to lung cancer was four months from the time of diagnosis.

The clinical suspicion of cardiac tamponade is crucial for the identification of a patient with malignant cardiac tamponade despite of non-specific symptoms and signs, because chest x-ray, ECG and even echochardiographic findings are not $100 \%$ sensitive or specific, as well. Pericardiocentesis provides diagnosis and offers this group of patients an immediate relief, but trials with various chemoterapeutic agents and radiotherapy, besides, a new surgical procedure will hopefully change the survival for this group of oncologic patients. ${ }^{12,13}$

\section{References}

1. Mukai K, Shinkai T, Tomonaga K, Shimoto Y. The incidence of secondary tumors of the heart and pericardium: A ten-year study. Jpn J Clin Oncol 1998; 18: 195-201.

2. Posner MR, Cohen GI, Skarin AT. Pericardial disease in patients with cancer. The differentiation of malignant from idiopathic and radiation-induced pericarditis. Am J Med 1981; 71: 407-13.

3. Fraser RS, Viloria JB, Wang N. Cardiac tamponade as a presentation of extra cardiac malignancy. Cancer 1980; 45: 1697-704.

4. Fincher E. Case Report: malignant pericardial effusion as the initial manifestation of malignancy. Am J med Sci 1993; 305: 106-10.

5. Muir KW, Rodger JC. Cardiac tamponade as the initial presentation of malignancy: is it as rare as previously supposed? Postcard Med J 1994; 70: 703-7.

6. Press OW, Livingston R. Management of malignant pericardial effusion and tamponade. JAMA 1987; 257: 1088-92.

7. Chong HH, Plotnick GD. Pericardial effusion and tamponade: evaluation, imaging, modalities, and management. Compr Ther 1995; 21: 378-85.

8. King DT, Nieberg RK. The use of cytology to evaluate pericardial effusions. Ann Clin Lab Sci 1979; 9: 18-23.

9. Wilding G, Green HL, Longo DL, Urba WJ. Tumors of the heart and pericardium. Cancer Treat Rev 1988; 5: 165-81.

10. Vaitkus PT, Herrmann HC, LeWinter MM. Treatment of malignant pericardial effusion. JAMA 1994; 272: 59-64.

11. Einama T, Sato $K$, Tsuda $H$, Mochizuki $H$. Successful treatment of malignant pericardial effusion, using weekly paclitaxel, in a patient with breast cancer. Int J Clin Oncol 2006; 11: 412-5.

12. Martinoni A. Treatment of neoplastic pericardial effusions. Recenti Prog Med 2006; 97: 206-10.

13. Tomkowski WZ, Wisniewska J, Szturmowicz M, Kuca P, Burakowski J, Kober J, et al. Evaluation of intrapericardial cisplatin administration in cases with recurrent malignant pericardial effusion and cardiac tamponade. Support Care Cancer 2004; 12: 53-7. 